\title{
Investigating fusion dynamics at high angular momentum via fission cross sections
}

\author{
C.S. Palshetkar ${ }^{1, \star}$, D.J. Hinde ${ }^{1, \star \star}$, E. Williams ${ }^{1}$, K. Ramachandran ${ }^{1, \star \star \star}$, M. Dasgupta ${ }^{1}$, K.J. Cook $^{1}$, A. Wakhle ${ }^{1}$, \\ D.Y. Jeung ${ }^{1}$, D.C. Rafferty ${ }^{1}$, S.D. McNeil ${ }^{1}$, I.P. Carter ${ }^{1}$, and D.H. Luong ${ }^{1}$ \\ ${ }^{1}$ Department of Nuclear Physics, Research School of Physical Sciences and Engineering, The Australian National University, \\ Canberra, ACT 2601, Australia
}

\begin{abstract}
A quantitative understanding of fusion dynamics at high angular momentum is attempted employing experimental fission cross sections as a probe and carrying out a simultaneous description of the fusion and fission cross sections at above barrier energies. For this, experimental fission fragment angular distributions for three systems: ${ }^{16} \mathrm{O}+{ }^{148} \mathrm{Sm},{ }^{28} \mathrm{Si}+{ }^{136} \mathrm{Ba}$ and ${ }^{40} \mathrm{Ca}+{ }^{124} \mathrm{Sn}$, all forming the same compound nucleus ${ }^{164} \mathrm{Yb}$ at similar excitation energies, have been measured at four beam energies above their respective capture barriers. A simultaneous description of the angle integrated fission cross sections and evaporation residue/fusion cross sections available in literature for the systems is carried out using coupled-channels and statistical model calculations. Fission cross sections, which are most sensitive to the changes in angular momentum, provide very stringent constraints for model calculations thus indicating the need of precision evaporation residue as well as fission cross sections in such studies. A large diffuseness $\left(\mathrm{a}_{o}>0.65 \mathrm{fm}\right)$ of the nuclear potential gives the best reproduction of the experimental data. In addition, different coupling schemes give very different angular momentum distributions, which, in turn, give very different fission cross section predictions. Both these observations hint at the explanation that depending on energy dissipation of the interacting nuclei occurring inside or outside the fusion pocket, very different fission cross sections can result due to heavily altered angular momentum and thus justifies the sensitivity of fission cross sections used as probes in the present work.
\end{abstract}

\section{Introduction}

Understanding fusion dynamics around the Coulomb barrier remains a topic of continued interest, particularly, due to a strong competition between compound and noncompound nuclear processes, altering the evolution of the compound nucleus $(\mathrm{CN})$ formed by heavy interacting nuclei. This is especially important in heavy element $(\mathrm{Z}>100)$ synthesis where these competing processes strongly suppress $\mathrm{CN}$ formation [1]. Another observation challenging our understanding of the fusion process has come from a systematic study of fusion cross section measurements for a large variety of projectile-target combinations [2]. Here, a large value of the diffuseness parameter of the Woods-Saxon form of the nuclear potential, $\mathrm{a}_{o} \sim 0.7-1.4 \mathrm{fm}$, was found to be required to reproduce the experimental fusion cross sections. This is in contrast to the value of $\mathrm{a}_{o}=0.65 \mathrm{fm}$ generally used for describing elastic scattering [3]. It was suggested in Ref. [2] that the large $\mathrm{a}_{o}$ values might be mocking up some dynamical effects not taken into account in model calculations for the fusion process.

\footnotetext{
^e-mail: asckj1@yahoo.co.in; Present Address: Department of Nuclear and Atomic Physics, Tata Institute of Fundamental Research, Mumbai- 400 005, India

$\star \star$ e-mail: David.Hinde@anu.edu.au

$\star \star \star$ Permanent Address: Nuclear Physics Division, Bhabha Atomic Research Center, Mumbai- 400 085, India
}

A large diffuseness results in a barrier shifted to lower radius values and a shallow fusion pocket [4]. In model calculations, fusion is generally described to occur when the interacting nuclei dissipate energy inside the fusion pocket where they are trapped, thus forming a CN. However, the system might dissipate energy not just inside but also outside the barrier. This might alter the flux of trajectories reaching inside the barrier, leading to reduced fusion cross sections and, as a result, different angular momentum $(l)$ distributions. Thus, the diffuseness parameter becomes an important parameter in such model calculations and the origin and implications of its large value should be investigated.

Dissipative effects are expected to be significant at higher bombarding energies and angular momenta. The effect of angular momentum in fusion could be probed by measuring the fission fragments, as fission is highly sensitive to the changes in angular momentum. This is because fission cross sections depend on the height of the fission barrier, which is angular momentum dependent. Previous works $[5,6]$ demonstrated a significant variation in the fission cross sections when different $l$ distributions were used in the model calculations. In this work, we test the sensitivity of fission cross sections on angular momentum by a systematic measurement of fission cross sections for the ${ }^{164} \mathrm{Yb} \mathrm{CN}$ populated through three different 
entrance channels, namely, ${ }^{16} \mathrm{O}+{ }^{148} \mathrm{Sm},{ }^{28} \mathrm{Si}+{ }^{136} \mathrm{Ba}$ and ${ }^{40} \mathrm{Ca}+{ }^{124} \mathrm{Sn}$. Fission fragment angular distributions have been measured for these systems at four beam energies above their respective Coulomb barriers. Simultaneous descriptions of fission cross sections from the present measurement and the evaporation residue/fusion cross sections available in the literature have been carried out using coupled channels and statistical model calculations.

\section{Experimental Details}

Fission fragment angular distributions were measured separately for the three systems using the 14UD electrostatic tandem accelerator at the Heavy Ion Accelerator Facility at the Australian National University, Canberra. Pulsed beams, separated by $107 \mathrm{~ns}$, of ${ }^{16} \mathrm{O}$ (laboratory beam en-

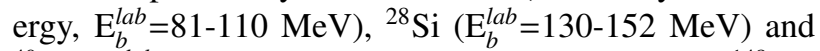
${ }^{40} \mathrm{Ca}\left(\mathrm{E}_{b}^{\text {lab }}=166-191 \mathrm{MeV}\right)$ were bombarded onto ${ }^{148} \mathrm{Sm}$ $\left(30 \mu \mathrm{g} / \mathrm{cm}^{2}+40 \mu \mathrm{g} / \mathrm{cm}^{2}{ }^{12} \mathrm{C}\right.$ backing $),{ }^{136} \mathrm{Ba}\left(40 \mu \mathrm{g} / \mathrm{cm}^{2}+\right.$ $12 \mu \mathrm{g} / \mathrm{cm}^{2}{ }^{12} \mathrm{C}$ backing $)$ and ${ }^{124} \mathrm{Sn}\left(17 \mu \mathrm{g} / \mathrm{cm}^{2}+12 \mu \mathrm{g} / \mathrm{cm}^{2}\right.$ ${ }^{12} \mathrm{C}$ backing) targets respectively, oriented at $\sim 30^{\circ}$ or $45^{\circ}$ with respect to the beam axis, at four beam energies above their respective Coulomb barriers. Charged particles arising from the reactions, namely, the fission fragments following fusion of the systems, elastically scattered beam particles and recoils were detected using the CUBE spectrometer [7] which consists of two large area Multi-Wire Proportional Counters (MWPC) with an active area of 27.9 $\mathrm{cm} \times 37.5 \mathrm{~cm}$ each. For all three measurements, one detector (MWPC 2) was placed at forward angle with respect to the beam axis, corresponding to the detector center at polar angle of $\theta_{2}=45^{\circ}$, giving an angular coverage from $5^{\circ}$ to $80^{\circ}$ and azimuthal angle of $\phi_{2}=0^{\circ}$. The other detector (MWPC 1) was placed at backward angles with respect to the beam axis, the angle depending on the expected folding angle of the fission fragments from each reaction, and with $\phi_{1}=180^{\circ}$. For the ${ }^{16} \mathrm{O}+{ }^{148} \mathrm{Sm}$ and ${ }^{28} \mathrm{Si}+{ }^{136} \mathrm{Ba}$ measurements, MWPC 1 was centered at $\theta_{1}=135^{\circ}$, giving an angular coverage from $95^{\circ}$ to $170^{\circ}$, while for the ${ }^{40} \mathrm{Ca}+{ }^{124} \mathrm{Sn}$ measurement, the detector center was at $\theta_{1}=90^{\circ}$, giving an angular coverage of $55^{\circ}$ to $130^{\circ}$. Both MWPCs were placed $18 \mathrm{~cm}$ away from the center of the target. Two silicon surface barrier detectors were also placed out of the reaction plane at $\theta_{m}^{l a b} \sim 23^{\circ}$ to serve as monitors.

Energy loss and time of flight signals with respect to the beam pulse from each detector were recorded on an event by event basis. The timing signals were taken from the central cathode foils of each MWPC. A 2D plot showing the raw timing signals from each MWPC obtained in the ${ }^{40} \mathrm{Ca}+{ }^{124} \mathrm{Sn}$ measurement is shown in Fig. 1 where the fission events are clearly distinguishable from the elastics and recoils. For measurements of the other two systems, only the fission events were collected in the coincidence gate due to the thresholds set in the electronics for rejection of elastics. The position information from each MWPC was obtained from the delay line readouts from both ends of the $\mathrm{X}$ and $\mathrm{Y}$ anode wires (1 mm apart). Signals from MWPC 2 were always recorded in coincidence with MWPC 1 to reduce the dead-time of the acquisition system. A pulser signal fed through the scaler and

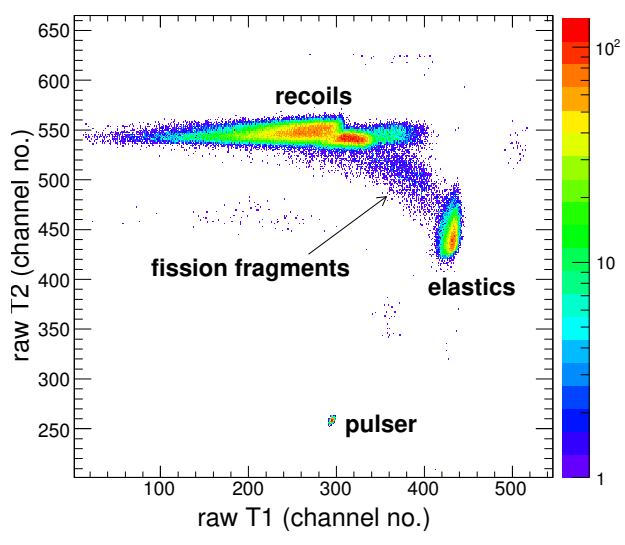

Figure 1. (Color online) Raw timing signals, from the central cathodes of the two MWPCs, recorded in the ${ }^{40} \mathrm{Ca}+{ }^{124} \mathrm{Sn}$ measurement.

data acquisition was used for the dead time corrections in the fission data. For the position calibration, events were recorded in singles mode, in which the entire active area of the detector gets illuminated. Thus, from the known positions, in $\mathrm{mm}$, of the detector edges, position calibration was done. Time calibration of the delay lines was done by inputting pulses separated by $10 \mathrm{~ns}$ from an ORTEC time calibrator into the delay lines and recording the output pulses. The solid angle normalization of the fission detectors was done using elastic scattering measured at beam energies much below the Coulomb barrier at $74.3 \mathrm{MeV}$ and $184.2 \mathrm{MeV}$ for the ${ }^{28} \mathrm{Si}+{ }^{197} \mathrm{Au}$ and the ${ }^{40} \mathrm{Ca}+{ }^{197} \mathrm{Au}$ reactions respectively.

\section{Analysis and Results}

The data analysis has three parts, (i) extracting the experimental fission cross sections from the measured angular distributions, (ii) coupled-channels calculations to get best reproduction of the experimental fusion cross sections and obtain the respective $l$-distributions (iii) using these $l$ distributions as input to statistical model calculations for fission cross section prediction.

\subsection{Experimental fission cross sections}

From the position information, the angles of the detected particles and from the timing information, their velocities were estimated. These velocities were then converted into masses using the kinematic coincidence technique described in the Appendix of Ref. [7]. By putting gates on the timing signals in Fig. 1, the fission events were clearly selected and yields corresponding to $5^{\circ}$ angle bins were extracted. These were corrected for solid angle to get the differential fission cross sections. The cross sections were converted from laboratory frame to the center of mass frame assuming symmetric binary fission and taking the kinetic energy of each fragment to be half of the total kinetic energy (TKE), where TKE is estimated from Viola systematics [8]. The experimental differential fission cross sections thus obtained are shown in Fig. 2 by filled points. 
The energies indicated in the figure which have been used while extracting the cross sections have been corrected for energy loss at the target center and converted to the center of mass frame. Due to limitations on the angular range covered with even such large area MWPCs, extrapolation of differential cross sections to $90^{\circ}$ and $180^{\circ}$ is required in order to extract the angle integrated fission cross sections. For this, the experimental differential cross sections were fitted with transition state model calculations for spin zero nuclei as done in Ref. [9] and are plotted in Fig. 2 as lines. The angle integrated fission cross sections thus obtained are given along with their errors in Fig. 2 and are plotted below in Fig. 4, 5 and 6 by filled squares.

\subsection{Coupled-channels calculations}

All coupled-channels (CC) calculations were carried out using the code CCFULL [10]. In this, it is necessary to constrain the potential parameters, the depth $\mathrm{V}_{o}$, radius parameter $\mathrm{r}_{o}$ and the diffuseness parameter $\mathrm{a}_{o}$. This was done as follows: (i) Starting with an initial set of $\mathrm{V}_{o}=200 \mathrm{MeV}$, $\mathrm{r}_{o}=1.0 \mathrm{fm}$ and $\mathrm{a}_{o}=1.0 \mathrm{fm}$, the parameters were varied iteratively to fit the experimental fusion cross sections for the systems taken from literature. In doing so, the uncoupled barriers quoted in literature for the systems, namely, $\mathrm{V}_{b}=59.8 \mathrm{MeV}$ [11] for ${ }^{16} \mathrm{O}+{ }^{148} \mathrm{Sm}$ and $\mathrm{V}_{b}=118.6 \mathrm{MeV}$ [12] for ${ }^{40} \mathrm{Ca}+{ }^{124} \mathrm{Sn}$, were also reproduced. (ii) Next, the experimental fusion barrier distributions were extracted from the fusion cross sections available in literature for each system using the three point difference method detailed in Ref. [13]. (iii) Inelastic states of the projectile and target were added one by one and checked iteratively to see coupling of which state gives a substantial change in fusion cross section predictions and also simultaneously fits the experimental cross sections as well as barrier distributions. Once a satisfactory fit to both was obtained, these couplings were used for final calculations and the potential parameters fine tuned. The best fit potential parameters for all three systems required $\mathrm{a}_{o}>0.65 \mathrm{fm}$. Another set of calculations keeping the best fit $\mathrm{V}_{o}$ and $\mathrm{a}_{o}=0.65 \mathrm{fm}$ was done where the $\mathrm{r}_{o}$ value was varied to get the same uncoupled barrier as the set with the larger $\mathrm{a}_{o}$. Thus, in the following, predictions of the $\mathrm{CC}$ and statistical model calculations are always shown for two sets of diffuseness, one with the fitted larger $\mathrm{a}_{o}$ and the other with $\mathrm{a}_{o}=0.65 \mathrm{fm}$.

As a representative of the above described procedure, in Fig. 3, are shown the experimental fusion cross sections for the ${ }^{16} \mathrm{O}+{ }^{148} \mathrm{Sm}$ system (filled circles) taken from [11, 14-16] plotted in the linear (Fig. 3(a)) and logarithmic scale (Fig. 3(b)) while the barrier distribution extracted from the data in the present analysis is plotted in Fig. 3(c).

For CC calculations, ${ }^{16} \mathrm{O}$ was considered inert while for ${ }^{148} \mathrm{Sm}$, a rotational coupling including static deformation with $\beta_{2}=0.18$ and $\beta_{4}=0.05$ was considered as done in [11]. In addition, a $3^{-}$vibrational state in ${ }^{148} \mathrm{Sm}$ (see Table 1 for details) was also coupled. The best fit potential parameters which simultaneously reproduce the experimental fusion cross sections, barrier distribution as well as the uncoupled barrier of $\mathrm{V}_{b}=59.8 \mathrm{MeV}$ [11] are
Table 1. Details of the vibrational states used in the CC calculations for the three systems studied in the present work. All the $\mathrm{B}(\mathrm{E} 2 \uparrow)$ have been taken from [17] and $\mathrm{B}(\mathrm{E} 3 \uparrow)$ values from [18].

\begin{tabular}{cccccc}
\hline \hline Nucleus & $\mathrm{J}^{\pi}$ & $\begin{array}{c}\mathrm{E}_{x} \\
(\mathrm{MeV})\end{array}$ & $\begin{array}{c}\mathrm{B}(\mathrm{E} \lambda \uparrow) \\
\left(\mathrm{e}^{2} \mathrm{~b}^{\lambda}\right)\end{array}$ & $\beta_{\lambda}$ \\
\hline \hline${ }^{148} \mathrm{Sm}$ & $3^{-}$ & 1.161 & 3 & 0.2911 & 0.1418 \\
${ }^{28} \mathrm{Si}$ & $3^{-}$ & 6.878 & 3 & 0.0042 & 0.4007 \\
${ }^{136} \mathrm{Ba}$ & $2^{+}$ & 0.818 & 2 & 0.410 & 0.1258 \\
& $3^{-}$ & 2.532 & 3 & 0.155 & 0.1253 \\
${ }^{40} \mathrm{Ca}$ & $3^{-}$ & 3.737 & 3 & 0.184 & 0.411 \\
${ }^{124} \mathrm{Sn}$ & $2^{+}$ & 1.132 & 2 & 0.1660 & 0.0953 \\
& $3^{-}$ & 2.603 & 3 & 0.073 & 0.1056 \\
\hline \hline
\end{tabular}

$\mathrm{V}_{o}=294 \mathrm{MeV}, \mathrm{r}_{o}=0.898 \mathrm{fm}$ and $\mathrm{a}_{o}=0.99 \mathrm{fm}$. For the set with $\mathrm{a}_{o}=0.65 \mathrm{fm}$ using the same $\mathrm{V}_{o}=294 \mathrm{MeV}, \mathrm{r}_{o}=1.076$ fm. From Fig. 3, it is clear that the larger diffuseness of $\mathrm{a}_{o}=0.99 \mathrm{fm}$ (solid line) gives the best fit to the experimental data as well as barrier distribution. The better agreement is especially clear at above barrier energies. The $l$ distributions obtained from above procedure were used for the statistical model calculations.

For the ${ }^{28} \mathrm{Si}+{ }^{136} \mathrm{Ba}$ calculations, due to the unavailability of fusion cross sections for the system, those corresponding to a nearby system, ${ }^{28} \mathrm{Si}+{ }^{142} \mathrm{Ce}$, taken from [19] were used. In the $\mathrm{CC}$ calculations, the $3^{-}$state of ${ }^{28} \mathrm{Si}$ and the $2^{+}$and $3^{-}$states in ${ }^{136} \mathrm{Ba}$, with the deformation parameters given in Table 1, were coupled as vibrational states. While comparing the $\mathrm{CC}$ results for ${ }^{28} \mathrm{Si}+{ }^{136} \mathrm{Ba}$ with the experimental ${ }^{28} \mathrm{Si}+{ }^{142} \mathrm{Ce}$ fusion cross sections, the geometrical effects were effectively accounted for using the reduction procedure described in [20], where $\sigma_{f u s} \rightarrow$ $\sigma_{f u s} /\left(\mathrm{A}_{p}^{1 / 3}+\mathrm{A}_{t}^{1 / 3}\right)^{2}$ and $\mathrm{E}_{c . m .} \rightarrow \mathrm{E}_{c . m .}\left(\mathrm{A}_{p}^{1 / 3}+\mathrm{A}_{t}^{1 / 3}\right) /\left(\mathrm{Z}_{p} \mathrm{Z}_{t}\right)$. Scaling factors for $\sigma_{f u s}$ of 0.0147 and for $\mathrm{E}_{c . m}$. of 0.0102 for the ${ }^{28} \mathrm{Si}+{ }^{142} \mathrm{Ce}$ and 0.0149 and 0.0104 respectively for ${ }^{28} \mathrm{Si}+{ }^{136} \mathrm{Ba}$ were thus obtained. The best fit potential parameters for this system are: $\mathrm{V}_{o}=194.5 \mathrm{MeV}, \mathrm{r}_{o}=0.971$ $\mathrm{fm}, \mathrm{a}_{o}=0.89 \mathrm{fm}$ and for $\mathrm{a}_{o}=0.65 \mathrm{fm}, \mathrm{V}_{o}=194.5 \mathrm{MeV}$ and $\mathrm{r}_{o}=1.064 \mathrm{fm}$.

A comparison of the $\mathrm{CC}$ results with the experimental fusion cross sections, showed that inelastic coupling alone was unable to reproduce the experimental cross sections, especially at below barrier energies (see Fig. 5 below). Thus, in addition to the inelastic states, transfer coupling was done for two different $Q$ values, the $2 n$ transfer with $\mathrm{Q}=3.002 \mathrm{MeV}$ and an effective $\mathrm{Q}$ value, corresponding to the $2 n+4 n$ transfer with $Q=5.133 \mathrm{MeV}$. These two sets of calculations were carried out to investigate which of them gave the biggest variation in the predicted cross sections. To account for the re-normalization of the barrier introduced due to transfer coupling, keeping $\mathrm{V}_{o}=194.5 \mathrm{MeV}$, the radius parameter was tweaked for the two different diffuseness. The strength parameter, $\mathrm{F}_{t}$, was varied in steps and a final value of $\mathrm{F}_{t}=0.5 \mathrm{MeV}$ was obtained which gave satisfactory reproduction of experimental fusion cross sections.

For the ${ }^{40} \mathrm{Ca}+{ }^{124} \mathrm{Sn}$ system, fusion evaporation cross sections were taken from $[12,21,22]$. Similar to the pro- 

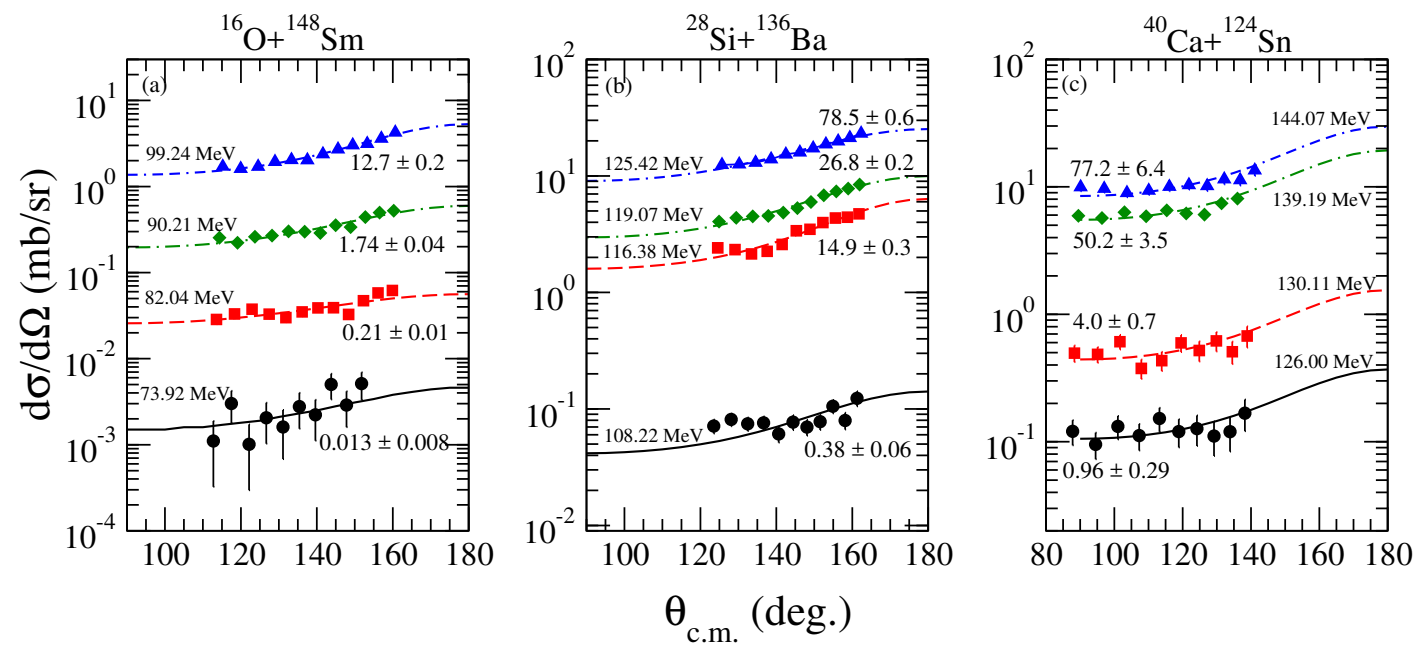

Figure 2. (Color online) Differential fission cross sections for (a) ${ }^{16} \mathrm{O}+{ }^{148} \mathrm{Sm}$, (b) ${ }^{28} \mathrm{Si}+{ }^{136} \mathrm{Ba}$ and (c) ${ }^{40} \mathrm{Ca}+{ }^{124} \mathrm{Sn}$ systems at four different energies in the center of mass frame. The values with errors are the angle integrated fission cross sections. The errors are statistical only.

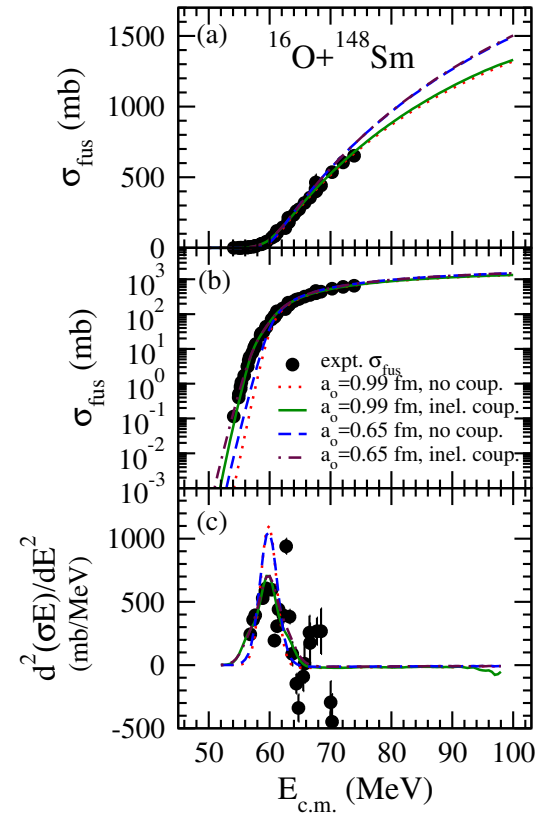

Figure 3. (Color online) Experimental fusion cross sections taken from [11, 14-16] (filled circles) plotted in the (a) linear and (b) logarithmic scale. The experimental barrier distribution extracted from the fusion data is plotted by the filled circles in (c). The dotted and dashed line are the results of the $\mathrm{CC}$ calculations without any coupling obtained by using $\mathrm{a}_{o}=0.99 \mathrm{fm}$ and $\mathrm{a}_{o}=0.65 \mathrm{fm}$ respectively. The solid line corresponds to the best fit obtained by including coupling of inelastic states and using $\mathrm{a}_{o}=0.99 \mathrm{fm}$ while the dot-dash line are CC results for the same coupling scheme but using $\mathrm{a}_{o}=0.65 \mathrm{fm}$.

cedure done for the other two system studied in the present work, the CC calculations for ${ }^{40} \mathrm{Ca}+{ }^{124} \mathrm{Sn}$ were done initially by coupling only the inelastic states, namely, the $3^{-}$ state in ${ }^{40} \mathrm{Ca}$ and the $2^{+}$and $3^{-}$states in ${ }^{124} \mathrm{Sn}$, included in the vibrational coupling mode. The best fit potential parameters in this case were found to be with $\mathrm{V}_{o}=200 \mathrm{MeV}$, $\mathrm{r}_{o}=0.984 \mathrm{fm}$ for $\mathrm{a}_{o}=1.0 \mathrm{fm}$ and $\mathrm{r}_{o}=1.107 \mathrm{fm}$ for $\mathrm{a}_{o}=0.65$ fm. Similar to the observations for ${ }^{28} \mathrm{Si}+{ }^{136} \mathrm{Ba}$ system, coupling of inelastic states alone did not reproduce the experimental cross sections. This is in line with the results obtained in $[12,21,22]$ where the importance of transfer coupling has been extensively shown. Hence, in the present analysis, calculations were performed with three different $\mathrm{Q}$ values: $\mathrm{Q}=3 \mathrm{MeV}, \mathrm{F}_{t}=0.5 \mathrm{MeV}, \mathrm{Q}=5.41 \mathrm{MeV}$ (2n transfer), $\mathrm{F}_{t}=0.7 \mathrm{MeV}$ and $\mathrm{Q}=9.5 \mathrm{MeV}$ (4n transfer), $\mathrm{F}_{t}=0.8 \mathrm{MeV}$.

\subsection{Statistical model fission calculations}

Statistical model calculations for the three systems were carried out using the code PACE [23]. The $l$-distributions, obtained from the CC calculations done using different coupling schemes (inelastic coupling only or inelastic + transfer with different $Q$ values), as described in sec 3.2, corresponding to the larger and smaller diffuseness of the potential, were given as input to PACE. The level density parameter at equilibrium deformation $\mathrm{a}_{n}$ was taken to be $\mathrm{a}_{n}=\mathrm{A} / 7.5$, following the semi-empirical formulation given in Equation A9 of Ref. [24]. The two other important adjustable parameters in PACE are, the ratio, $\mathrm{a}_{f} / \mathrm{a}_{n}$, where $\mathrm{a}_{f}$ is the level density at the saddle point and the scaling factor, $\mathrm{k}$, for the fission barrier. For estimating $\mathrm{k}$, using $\mathrm{a}_{f} / \mathrm{a}_{n}=1.00$, for the ${ }^{16} \mathrm{O}+{ }^{148} \mathrm{Sm}$ system, $\mathrm{k}$ was varied in steps and for each $\mathrm{k}$, the $\chi^{2}$ of the fit of the resultant fission cross sections to the experimental values measured in the present work was determined. In this way, the $\mathrm{k}$ giving the minimum $\chi^{2}, \mathrm{k}=0.997$ for $\mathrm{a}_{o}=0.99 \mathrm{fm}$ and $\mathrm{k}=1.084$ for $\mathrm{a}_{o}=0.65 \mathrm{fm}$ was obtained and used for the final calculations. This procedure was also carried out using $\mathrm{a}_{f} / \mathrm{a}_{n}=1.04$ and although different values of $\mathrm{k}$ were obtained, the resultant fission cross sections were very similar. Hence, in the following, results using only $\mathrm{a}_{f} / \mathrm{a}_{n}=1.00$ are shown.

The results of the simultaneous analysis of fusion and fission cross sections for the ${ }^{16} \mathrm{O}+{ }^{148} \mathrm{Sm}$ are shown in 


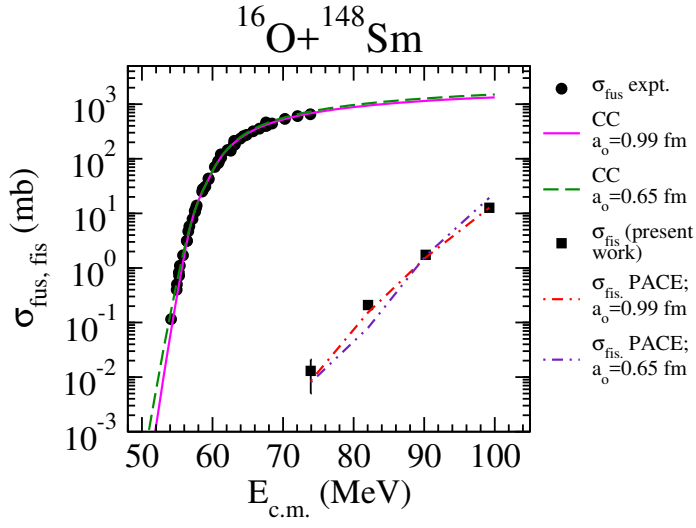

Figure 4. (Color online) Comparison of the experimental fusion $[11,14-16]$ (filled circles) and fission (filled squares) cross sections from the present work with the predictions of the statistical model calculations PACE. The solid and dashed line are the CC results obtained by coupling the inelastic states in the projectile and target (same inel. coup. results as plotted in Fig. 3 by solid and dot-dash lines) for the two different diffuseness parameters respectively. The corresponding fission cross sections estimated from PACE are plotted as dot-dash line for $\mathrm{a}_{o}=0.99 \mathrm{fm}$ and dotdot-dash lines for $\mathrm{a}_{o}=0.65 \mathrm{fm}$.

Fig. 4. The beam energy dependence of the fission calculations from PACE, corresponding to the larger diffuseness parameter, $\mathrm{a}_{o}=0.99 \mathrm{fm}$ (dot-dash line) are in an overall better agreement with the experimental cross sections.

Taking the same $\mathrm{k}$ values as for ${ }^{16} \mathrm{O}+{ }^{148} \mathrm{Sm}$ system and $\mathrm{a}_{f} / \mathrm{a}_{n}=1.00$, PACE calculations were carried out for the other two systems as well. Results for the ${ }^{28} \mathrm{Si}+{ }^{136} \mathrm{Ba}$ are shown in Fig. 5. Fission predictions obtained by inputting the $l$-distributions corresponding to inelastic states only (Fig. 5(a)) is in disagreement with the experimental values, even though the experimental fusion cross sections at above barrier energies are reproduced satisfactorily. Results corresponding to inelastic + transfer, shown in Fig. 5(b) and (c) are well reproduced. Here again, though the difference in the predictions for the two diffuseness values is small, predictions corresponding to $\mathrm{a}_{o}=0.65$ fm show a steeper fall with decreasing energy as compared to those for $\mathrm{a}_{o}=0.89 \mathrm{fm}$ and poorly reproduce the experimental cross sections. Effect of different transfer $Q$ values used for calculations is not significant.

Results for ${ }^{40} \mathrm{Ca}+{ }^{124} \mathrm{Sn}$ system are shown in Fig. 6. As for the other two systems shown above, the best fission predictions from PACE are obtained for inelastic + transfer coupling $(\mathrm{Q}=3 \mathrm{MeV})$ and once again the larger diffuseness gives better predictions. As the transfer $Q$ value is increased, the corresponding $l$-distributions are also altered giving very different fission predictions. This shows the sensitivity of the fission cross sections to the type of couplings used for the calculations.

\section{Summary and Conclusions}

To summarize, this work focuses on investigating the sensitivity of fission cross sections in understanding the fusion

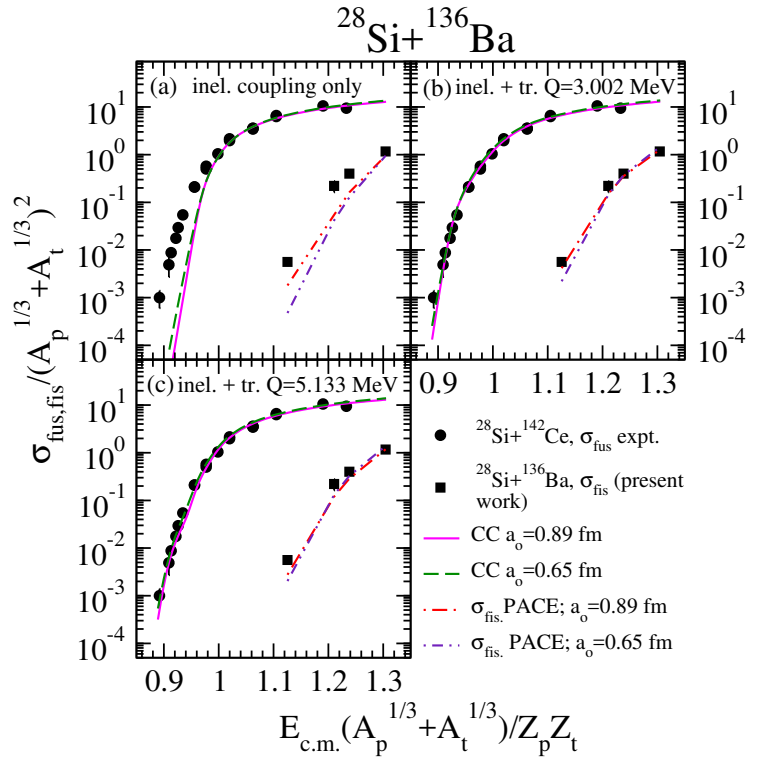

Figure 5. (Color online) Similar to Fig. 4 but for ${ }^{28} \mathrm{Si}+{ }^{136} \mathrm{Ba}$ system. The reduced cross sections have been plotted in order to show the experimental fusion cross sections for ${ }^{28} \mathrm{Si}+{ }^{142} \mathrm{Ce}$ [19] (filled circles) with predictions of model calculations for the ${ }^{28} \mathrm{Si}+{ }^{136} \mathrm{Ba}$ system. Results from PACE when $l$-distributions extracted from inelastic coupling only are used for the input in the calculations are plotted in (a). (b) shows the results for inelastic + transfer with $\mathrm{Q}=3.002 \mathrm{MeV}$ while (c) shows the results for inelastic + transfer with $\mathrm{Q}=5.133 \mathrm{MeV}$.

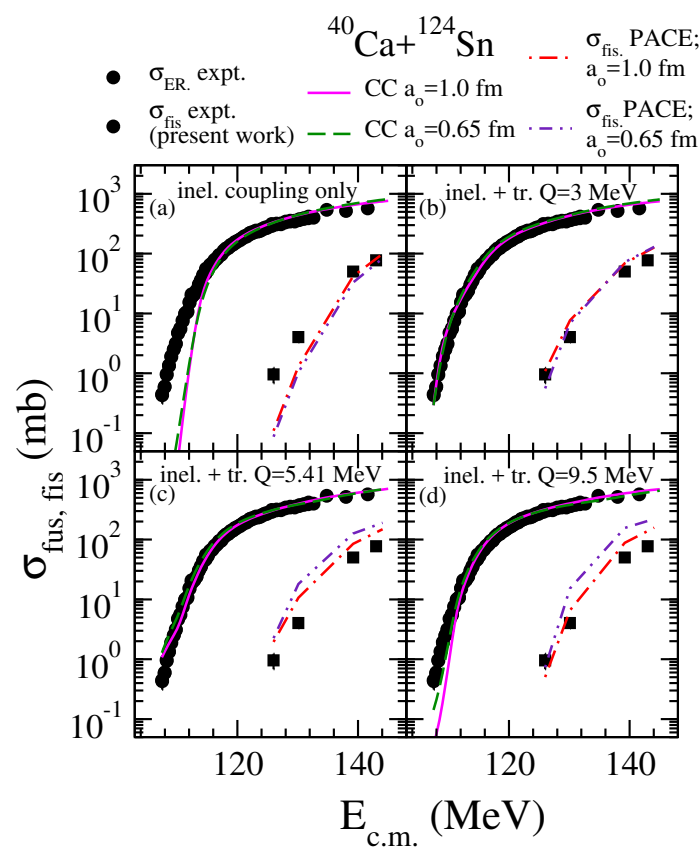

Figure 6. (Color online) Similar to Fig. 4 and 5 but for ${ }^{40} \mathrm{Ca}+{ }^{124} \mathrm{Sn}$ system. Results from PACE corresponding to $l$ distributions obtained from inelastic coupling only are plotted in (a) whereas those corresponding to results from inelastic + transfer coupling are plotted in (b) for transfer $\mathrm{Q}=3 \mathrm{MeV}$, in (c) for $\mathrm{Q}=5.41 \mathrm{MeV}$ and in (d) for $\mathrm{Q}=9.5 \mathrm{MeV}$. 
dynamics at high angular momentum. For this, measurement of fission cross sections for the $\mathrm{CN}{ }^{164} \mathrm{Yb}$, formed via different entrance channels has been carried out. A simultaneous description of the fusion as well as fission cross sections has been done using the $\mathrm{CC}$ and statistical model calculations.

Stringent constraints are put on model predictions by requiring a simultaneous reproduction of fusion as well as fission cross sections over the entire energy range of the available data. The fission cross sections are observed to be very sensitive to the shape of the $l$-distributions as well as the type of couplings used in the model calculations. With increasing projectile charge, the importance of transfer channels is seen, which again alters the $l$-distributions. A larger diffuseness of the nuclear potential used in the CC calculations and the $l$-distributions resulting from the same is required for the best reproduction of the experimental fission cross sections measured in the present work, in line with previous observations [2]. The present results provide support to the explanation for the requirement of the large diffuseness to be due to energy dissipation of the system possibly occurring outside the potential pocket thus altering the flux of trajectories and ultimately the fusion cross section. The present work emphasizes the requirement of precision absolute evaporation residue and as well as fission cross sections and the procedure of simultaneous analysis to understand the fusion process at high angular momentum.

\section{Acknowledgments}

The authors thank the accelerator and technical staff of the Heavy Ion Accelerator Facility at the Australian National University for their help during the experiment.

\section{References}

[1] D.J. Hinde and M. Dasgupta, Nucl. Phys. A 787, 176c (2007).

[2] J.O. Newton, R.D. Butt, M. Dasgupta, D.J. Hinde, I.I. Gontchar, C.R. Morton and K. Hagino, Phys. Rev. C 70, 024605 (2004).

[3] R.O. Akyuz, and A. Winther, in Proc. Enrico Fermi. Int. School of Physics edited by R.A. Broglia, C.H. Dasso and R. Richi North-Holland, Amsterdam, (1981).

[4] M. Dasgupta, D.J. Hinde, J.O. Newton and K. Hagino, Prog. Theo. Phys. Supp. 154, 209 (2004).

[5] R.J. Charity, J.R. Leigh, J.J.M. Bokhorst, A. Chatterjee, G.S. Foote, D.J. Hinde, J.O. Newton, S. Ogaza and D. Ward, Nucl. Phys. A 457, 441 (1986).

[6] Y. Kondo, B.A. Robson, J.J.M. Bokhorst, D.J. Hinde and J.R. Leigh, Phys. Rev. C 35, 828 (1987).
[7] D.J. Hinde, M. Dasgupta, J.R. Leigh, J.C. Mein, C.R. Morton, J.O. Newton and H. Timmers, Phys. Rev. C 53, 1290 (1996).

[8] V.E. Viola, K. Kwiatkowski and M. Walker, Phys. Rev. C 31, 1550 (1985).

[9] B.B. Back, R.R. Betts, J.E. Gindler, B.D. Wilkins, S. Saini, M.B. Tsang, C.K. Gelbke, W.G. Lynch, M.A. McMahan and P.A. Baisden, Phys. Rev. C 32, 195 (1985).

[10] K. Hagino, N. Rowley and A.T. Kruppa, Comp. Phys. Comm. 123, 143 (1999).

[11] J.R. Leigh, M. Dasgupta, D.J. Hinde, J.C. Mein, C.R. Morton, R.C. Lemmon, J.P. Lestone, J.O. Newton, H. Timmers and N. Rowley, Phys. Rev. C 52, 3151 (1995).

[12] A.M. Stefanini, L. Corradi, D. Ackermann, C.J. Lin, L.F. Zheng, S. Beghini, G. Montagnoli, F. Scarlassara and G.F. Segato, IL Nuovo Cimento 111, 895 (1998).

[13] M. Dasgupta, D.J. Hinde, N. Rowley and A.M. Stefanini, Annu. Rev. Nucl. Part. Sci. 48, 401 (1998).

[14] R.G. Stokstad, Y. Eisen, S. Kaplanis, D. Pelte, U. Smilansky and I. Tserruya, Phys. Rev. Lett. 41, 465 (1978).

[15] R.G. Stokstad, Y. Eisen, S. Kaplanis, D. Pelte, U. Smilansky and I. Tserruya, Phys. Rev. C 21, 2427 (1980).

[16] D.E. DiGregorio, M. diTada, D. Abriola, M. Elgue, A. Etchegoyen, M.C. Etchegoyen, J.O Fernández Niello, A.M.J. Ferrero, S. Gil, A.O. Macchiavelli, A.J. Pacheco, J.E. Testoni, P.R.Silveira Gomes, V.R. Vanin, R. Liguori Neto, E. Crema and R.G. Stokstad, Phys. Rev. C 39, 516 (1989).

[17] S. Raman, C.W. Nestor, JR., and P. Tikkanen, Atomic Data and Nuclear Data Tables 78, 1 (2001).

[18] T. Kibédi and R.H. Spear, Atomic Data and Nuclear Data Tables 80, 35 (2002).

[19] S. Gil, F. Hasenbalg, J.E. Testoni, D. Abriola, M.C. Berisso, M. di Tada, A. Etchegoyen, J.O. Fernández Niello, A.J. Pacheco, A. Charlop, A.A. Sonzogni and R. Vandenbosch, Phys. Rev. C 51, 1336 (1995).

[20] P.R.S. Gomes, J. Lubian, I. Padron and R.M. Anjos, Phys. Rev. C 71, 017601 (2005).

[21] F. Scarlassara, S. Beghini, G. Montagnoli, G.F. Segato, D. Ackermann, L. Corradi, C.J. Lin, A.M. Stefanini and L.F. Zheng, Nucl. Phys. A 672, 99 (2000).

[22] J.J. Kolata, A. Roberts, A.M. Howard, D. Shapira, J.F. Liang, C.J. Gross, R.L. Varner, Z. Kohley, A.N. Villano, H. Amro, W. Loveland and E. Chavez, Phys. Rev. C 85, 054603 (2012).

[23] A. Gavron, Phys. Rev. C 21, 230 (1980).

[24] J. Tōke and W.J. Świa̧tecki, Nucl. Phys. A 372, 141 (1981). 